Check for updates

Cite this: RSC Adv., 2017, 7, 36175

\title{
Fabrication of reactive pigment composite particles for blue-light curable inkjet printing of textiles
}

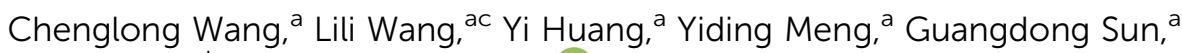 \\ Qinguo Fan ${ }^{\mathrm{ab}}$ and Jianzhong Shao (D) *a
}

\begin{abstract}
The reactive pigment composite particles were fabricated by a two-step method. Phthalocyanine Blue (PB) was encapsulated with silica through a Sol-Gel method in the first step, and the encapsulation conditions were optimized. 3-(Trimethoxysilyl)propyl methacrylate was then grafted onto the silica encapsulated organic pigment (EPB) in the second step, and the FT-IR analysis indicated that the relevant functional groups had been introduced onto the EPB. The particle size, hydrophilicity and the color characteristics of the Reactive Phthalocyanine Blue (RPB) changed little in comparison with PB. The photopolymerization performance of the RPB-based ink showed a certain increase, and the fastness toward crocking of the printed fabrics was coordinately improved.
\end{abstract}

Received 24th April 2017

Accepted 13th July 2017

DOI: 10.1039/c7ra04576j

rsc.li/rsc-advances

particles onto surface of organic pigment. Recently, several methods have been reported for the encapsulation of pigment particles such as emulsion or mini-emulsion polymerization, ${ }^{\mathbf{1 6}, 17}$ phase separation, ${ }^{\mathbf{1 8 , 1 9}}$ layer by-layer assembly, ${ }^{20}$ and sol-gel method. ${ }^{21,22}$ Nevertheless, the encapsulated pigments by these methods were still lack of firm forces in the crosslinking film. In our previous work, ${ }^{23}$ reactive organic pigment complex was prepared by the absorption-encapsulation of 3(trimethoxysilyl)propyl methacrylate (MPS) onto the surface of organic pigment. However, the surface of $\mathrm{PB}$ was lack of reactive groups, so that the distribution of MPS onto PB was difficult to be controlled effectively. Thus, the hydrophilicity of reactive organic pigment complex were slightly increased, resulting in the increase of the particle size in ink. Silica was considered to be a good candidate of the transition layer, because it was convenient to coat on organic pigment and easy to be functionalized. ${ }^{24-26}$ After encapsulation of silica, hydroxyl group can be induced onto the surface of $\mathrm{PB}$, which has good reactivity with various coupling agents. ${ }^{27,28}$ Based on the above idea, two-step method was proposed to prepare reactive pigment composite particles.

In this paper, silica encapsulated organic pigment (EPB) was firstly prepared by Sol-gel method, and the Reactive Phthalocyanine Blue (RPB) was then prepared by grafting MPS onto the surface of EPB. The double bonds existed in RPB can participate in the copolymerization of oligomers and monomers to realize the firm anchorage of pigment particles in the polymer crosslinked network structure via covalent bonds. The optimum process to prepare EPB was investigated. The particle size, color performance and hydrophilicity of RPB were studied. The application of the RPB in blue light curable inks was carried out and the colorfastness toward crocking of the printed fabric was investigated.

\footnotetext{
${ }^{a}$ Engineering Research Center for Eco-Dyeing and Finishing of Textiles, Ministry of Education, Zhejiang Sci-Tech University, Hangzhou 310018, China.E-mail: jshao@ zstu.edu.cn; Fax: +86-571-86843666; Tel: +86-571-86843625

${ }^{b}$ Department of Bioengineering, University of Massachusetts Dartmouth, North Dartmouth, MA 02747, USA

'Saintyear Holding Group Co., Ltd., Hangzhou 311221, China
} 


\section{Experimental sections}

\subsection{Materials}

Poly(diallyldimethylammonium chloride) (PDADMAC, Mw < 200 000), 2-hydroxyethylacrylate (HEA) and 3-(trimethoxysilyl) propyl methacrylate (MPS) were obtained from Aladdin Industrial Corporation. Ammonia $\left(\mathrm{NH}_{3}\right)$ and absolute ethanol were purchased from Hangzhou Gaojing Fine Chemical Co. Ltd. Poly(sodium 4-styrenesulfonate) (PSS, Mw = 70 000) was obtained from Shanghai Macklin Biochemical Co. Ltd. Polyvinylpyrrolidone (PVP) was obtained from Tianjin Kemiou Chemical Reagent Co. Ltd. Camphorquinone (CQ), ethyl-4dimethylaminobenzoate (EDMAB) and tetraethyl orthosilicate (TEOS) were obtained from Sigma-Aldrich Co. LLC. Phthalocyanine Blue was provided from Clariant Chemical Co. Ltd. A commercial oligomer SM6202 was supplied by Jiangsu Sanmu Group Corporation.

\subsection{Preparation of EPB composite particles}

$1 \mathrm{~g}$ Phthalocyanine Blue pigment was added into $0.5 \mathrm{~g} \mathrm{~L}^{-1}$ PSS solution. The mixture was milled by the planetary ball mill (PM400, Germany) with $6 \mathrm{~mm}$ stainless steel ball and dispersed for $1 \mathrm{~h}$ at $300 \mathrm{rpm}$, centrifugal washing to remove the residual PSS. Then, the above organic pigment was added to $1 \mathrm{~g} \mathrm{~L}^{-1}$ PDADMAC solution (containing $0.3 \mathrm{~mol} \mathrm{~L}^{-1} \mathrm{NaCl}$ ). After adsorption for $30 \mathrm{~min}$, centrifugal washing to remove the residual PDADMAC. The organic pigment with positive charges was redispersed into a certain amount of deionized water and $200 \mathrm{~mL}$ ethanol. $0.2 \mathrm{~g}$ PVP was sequentially added and $\mathrm{pH}$ was adjusted. A certain amount of TEOS and $40 \mathrm{~mL}$ ethanol were dropped into the organic pigment dispersion solution within $1 \mathrm{~h}$. After that, the reactant was stirred for $20 \mathrm{~h}$ at $25{ }^{\circ} \mathrm{C}$. Finally, centrifugal washing and dried. The preparation route of EPB was shown in Scheme 1.

\subsection{Preparation of reactive pigment composite particles}

Reactive pigment composite particles were achieved by the following procedure: $1 \mathrm{~g}$ EPB was dispersed into $100 \mathrm{~mL}$ solution of ethanol/water $(2 / 8, v / v)$. The dispersion was ultrasonically dispersed for $30 \mathrm{~min}$, then a solution of $0.1 \mathrm{~g}$ MPS in $20 \mathrm{~mL}$ solution of ethanol/water $(2 / 8, \mathrm{v} / \mathrm{v})$ was added into the above dispersion. And the mixture was stirred for $3 \mathrm{~h}$ further at $75^{\circ} \mathrm{C}$. The obtained sample was rinsed twice with deionized water and dried at $40{ }^{\circ} \mathrm{C}$ for $24 \mathrm{~h}$. The schematic mechanism of preparation of reactive pigment composite particles was shown in Scheme 2.

\subsection{Preparation of the blue light curable inks}

A blue light curable ink was composed of 1 wt $\%$ CQ/EDMAB combination as photoinitiators, a commercial oligomer/HEA/ IBOA combination as medium, $1.0 \mathrm{wt} \%$ as-prepared reactive pigment or $\mathrm{PB}$. The mixture was dispersed by a covered ultrasonic oscillator ( $250 \mathrm{~W}, 40 \mathrm{kHz}$, Kunshan Ultrasound Co., Ltd).

\subsection{Photo-differential scanning calorimetry measurement}

The photo-polymerization behavior of as-prepared blue light curable ink was examined by photo-DSC analysis. Photo-DSC measurements were performed using a differential scanning calorimeter (Q2000, TA Instruments, USA) equipped with a photo-calorimetric accessory (PCA OmniCure S2000, Canada).

\subsection{BET analysis}

Nitrogen adsorption/desorption isotherms were measured using a Micromeritics ASAP 2020HD88. The Brunauer-EmmettTeller (BET) method was used to analyze the specific surface areas of the samples.

\subsection{FT-IR analysis}

Fourier transform infrared (FT-IR) spectra of the samples was recorded on a FT-IR spectrometer (Nicolet 5700, USA) at a resolution of $4 \mathrm{~cm}^{-1}$ in the range from 4000 to $600 \mathrm{~cm}^{-1}$.

\subsection{TEM analysis}

The morphologies of PB, EPB and RPB were taken using a JSM2100 transmission electron microscope (JEOL, Japan) at an acceleration voltage of $200 \mathrm{kV}$.

\subsection{Particle size analysis}

The samples of PB and EPB were prepared respectively by mixing pigment $(0.01 \mathrm{~g})$ and deionized water $(10 \mathrm{~g})$, which were subjected to ultrasonic treatment for $30 \mathrm{~min}$, and then diluted in same times. The particle sizes of the samples were determined by a Malvern laser particle sizes analyzer (Nano-S, England).
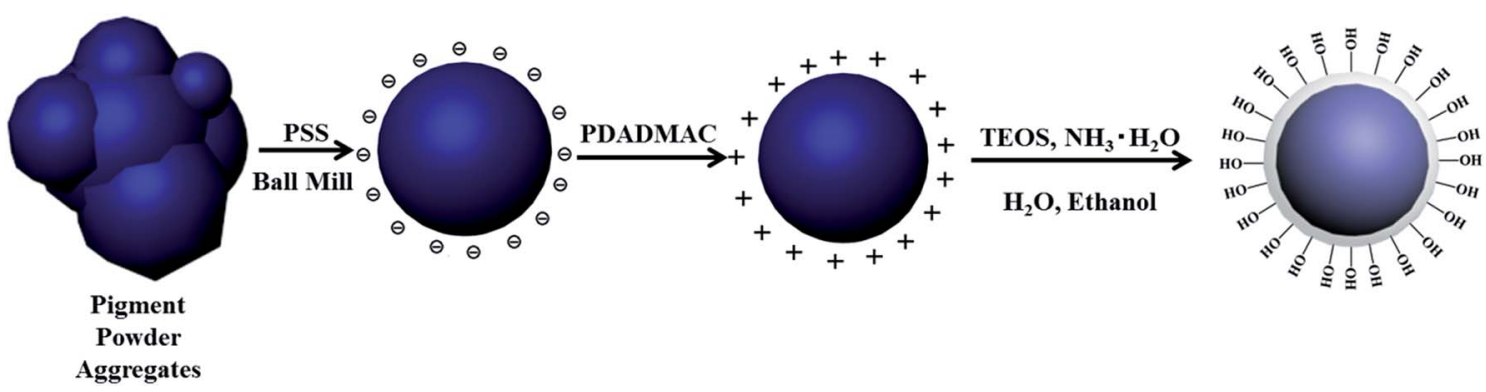

Scheme 1 Schematic diagram for the preparation of EPB composite particles. 

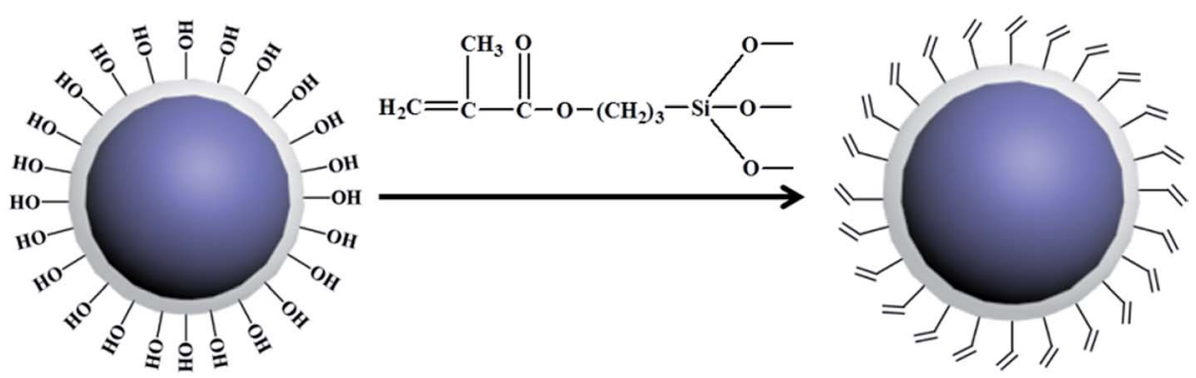

Scheme 2 Mechanism for preparation of RPB.

The samples of $\mathrm{PB}$ and RPB were prepared respectively by mixing organic pigment $(0.01 \mathrm{~g})$ and HEA $(10 \mathrm{~g})$, which were subjected to ultrasonic treatment for $30 \mathrm{~min}$, and then diluted in same times. The particle sizes of the samples were determined by a Malvern laser particle sizes analyzer (Nano-S, England).

\subsection{Dispersion stability}

The samples of PB and RPB were prepared respectively by mixing pigment $(0.01 \mathrm{~g})$ and HEA $(10 \mathrm{~g})$, which were subjected to ultrasonic treatment for $30 \mathrm{~min}$. After standing for a week, the digital photographs were taken by digital camera (Sony).

\subsection{Surface tension}

The surface tension of inks was measured by using a contactangle system (DSA-20, Germany) at ambient temperature and saturated humidity.

\subsection{Thermogravimetric analyses (TGA)}

EPB and PB were analyzed for their thermal behavior using a Perkinelmer Pyris 1 TGA thermal analysis instrument. The samples were heated from 25 to $700^{\circ} \mathrm{C}$ at a heating rate of $20^{\circ} \mathrm{C}$ $\min ^{-1}$ under $\mathrm{N}_{2}$ atmosphere.

\subsection{UV-vis spectrophotometer}

The reflection of PB and RPB was detected on a UV-vis spectrophotometer (UV-2600, Japan) using the diffuse reflectance mode.

\subsection{Digital printing of cotton fabrics}

A digital printing trial using the as-prepared blue light curable ink was carried out on a plain woven cotton fabric with fluid dispensing equipment (TenSun TS-500B, China) at $20 \mathrm{~mm} \mathrm{~s}^{-1}$ moving speed with $140 \mathrm{kPa}$ nitrogen pressures. After printing, the printed fabrics were cured under a self-assembled blue light emitting diode (LED) lamp in nitrogen atmosphere for $5 \mathrm{~min}$.

\subsection{Measurement of rubbing color-fastness}

The dry and wet rubbing color-fastness of the printed cotton fabrics was measured by employing a rubbing tester (680MD, UK) according to AATCC 8-2007 test method.

\subsection{Mechanical properties}

The cured films were prepared by blue light curable ink. The elongation at break and breaking strength of cured films were tested by Instron 3367 material testing machine according to ASTM D638-2014 standard.

\subsection{Handle analysis}

The handle of printed fabric was tested by intelligent fabric style instrument (YG821LPhabrOmeterfabric, China), and the samples were cut into circle with diameter of $12 \mathrm{~cm}$.

\section{Results and discussion}

\subsection{Preparation of EPB}

The effective encapsulation of organic pigment with silica was beneficial for the preparation of RPB. The encapsulation of organic pigment via sol-gel process of TEOS was carried out in ethanol at room temperature. The effects of deionized water, $\mathrm{pH}$ and TEOS on the preparation of EPB were investigated.

3.1.1 Effect of deionized water on the preparation of EPB. The influences of deionized water on the encapsulation of organic pigment with silica are shown in Table 1 . The particle size and PDI of PB had the biggest value. After silica encapsulation, the particle size and PDI of EPB decreased. This was probably because organic pigment was encapsulated by silica, so that the hydrophilicity and the dispersion stability of EPB were improved. The particle size and PDI of EPB increased with increasing of deionized water. It might be that high water content could result in fast hydrolysis and condensation of TEOS, accompanied by the strong tendency to self-nucleation. Therefore, the optimum dosage of deionized water was $1: 16$ $\left(V_{\text {water }} / V_{\text {ethanol }}\right)$.

3.1.2 Effect of $\mathbf{p H}$ on the preparation of EPB. Table 2 displays the effects of $\mathrm{pH}$ on the encapsulation of organic pigment with silica. In Table 2, when $\mathrm{pH}$ values were 11 and 12,

Table 1 Particle size and PDI of PB and EPB with different water concentration

\begin{tabular}{lccccc}
\hline & PB & $1: 20$ & $1: 16$ & $1: 12$ & $1: 10$ \\
\hline Z-Aver & 404.8 & 253.0 & 222.0 & 278.8 & 301.2 \\
PDI & 0.419 & 0.208 & 0.209 & 0.269 & 0.327
\end{tabular}


Table 2 Particle size and PDI of EPB with different $\mathrm{pH}$

\begin{tabular}{lcccc}
\hline & 9 & 10 & 11 & 12 \\
\hline Z-Aver & 261.2 & 222.0 & 333.8 & 374.5 \\
PDI & 0.296 & 0.209 & 0.320 & 0.376
\end{tabular}

Table 3 The specific surface areas of PB and EPB with different $\mathrm{pH}$

\begin{tabular}{lll}
\hline Sample & $\begin{array}{l}\text { BET surface } \\
\text { area }\left(\mathrm{m}^{2} \mathrm{~g}^{-1}\right)\end{array}$ & $\begin{array}{l}\text { Langmuir surface } \\
\text { area }\left(\mathrm{m}^{2} \mathrm{~g}^{-1}\right)\end{array}$ \\
\hline $\mathrm{PB}$ & 50.57 & 81.15 \\
$\mathrm{pH}=9$ & 51.56 & 82.17 \\
$\mathrm{pH}=10$ & 55.45 & 89.28 \\
$\mathrm{pH}=11$ & 73.83 & 118.11 \\
$\mathrm{pH}=12$ & 68.82 & 109.76
\end{tabular}

the particle sizes and PDI were obviously increased. It might be attributed to high $\mathrm{pH}$ value, leading to the fast hydrolysis and condensation of TEOS.

The specific surface areas of the PB particles and EPB with different $\mathrm{pH}$ were determined by BET and Langmuir method and listed in Table 3 . It could be found that the specific surface area of EPB had little change at $\mathrm{pH}$ value 9 and 10. However, at $\mathrm{pH} 11$ and 12, the specific surface of EPB were obviously increased. In Fig. 1, it also demonstrated that the $\mathrm{N}_{2}$ adsorption/desorption isotherms of EPB were similar to that of the $\mathrm{PB}$ particles at $\mathrm{pH} 9$ and 10 . When relative pressure $P / P_{0}$ was more than 0.75 , a slight separation of the adsorption isotherms and desorption isotherms of EPB was also observed at $\mathrm{pH} 11$ and 12, which should be the evidence of a small quantity of micropores existed on the organic pigment surfaces, due to the generation of $\mathrm{SiO}_{2}$ nanoparticles on the surface of organic pigment. ${ }^{21}$ As a result, the optimum pH value was 10.
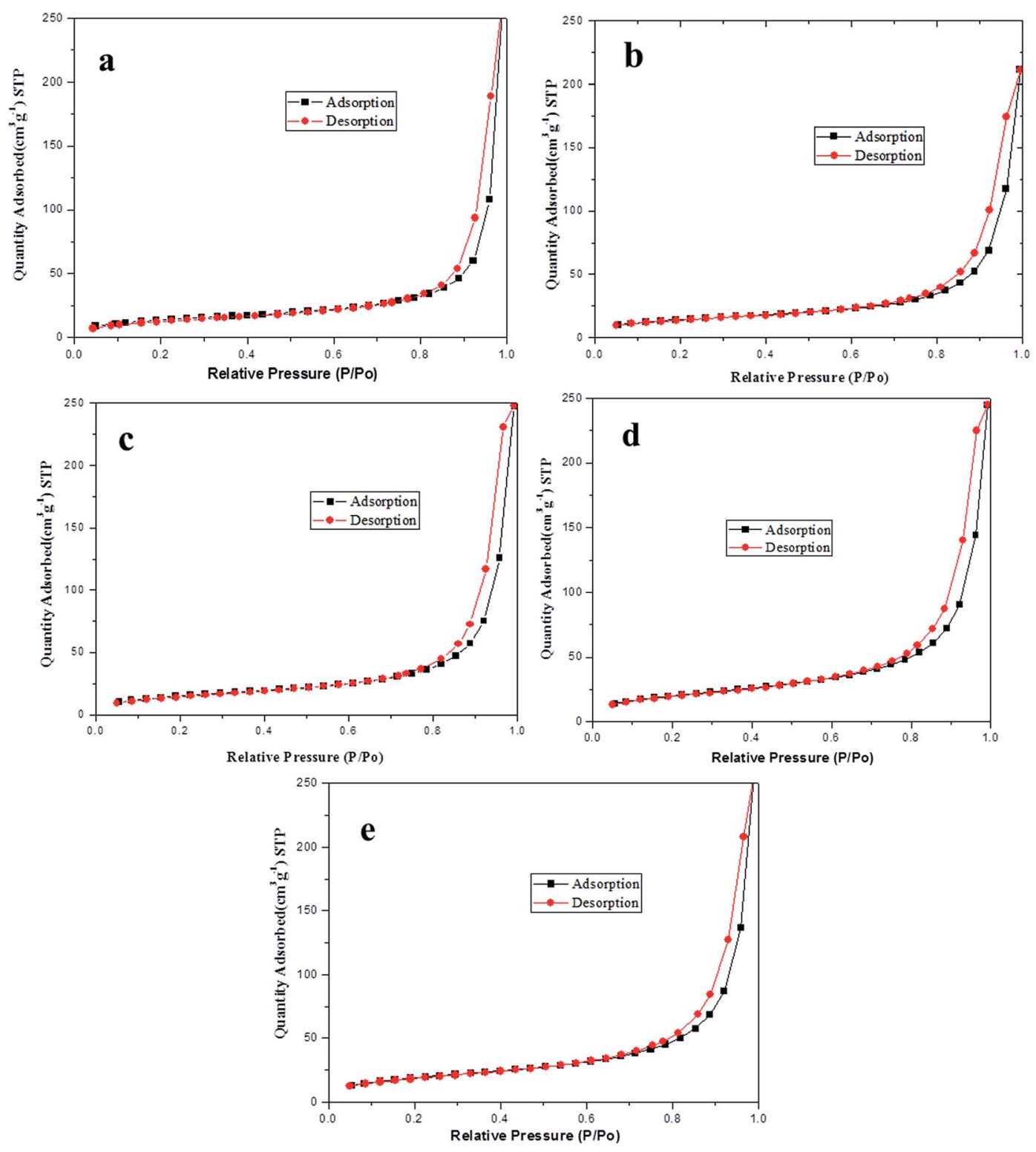

Fig. 1 Nitrogen adsorption/desorption isotherm of EPB with different $p H$ value (a: $P B, b: p H=9, c: p H=10, d: p H=11, e: p H=12)$. 
3.1.3 Effect of TEOS on the preparation of EPB. Table 4 shows the effect of TEOS dosage on the encapsulation of organic pigment. When the concentration of TEOS changed, the particle size and PDI of EPB had little change. Furthermore, the amount of silica, which was encapsulated on the surface of organic pigment, was characterized by TGA, shown in Fig. 2. As TEOS increased, the amount of silica encapsulate on the surface of organic pigment increased. It also implied that the content of silica encapsulated on the surface of organic pigment could be easily modulated with various TEOS dosage. Fig. 2 shows the thermogravimetric results of the PB and EPB and Fig. 3 gives the differential of the TGA curves in Fig. 2. It was found that the PB had two stages of weight loss while EPB had three stages. It demonstrated that the thermostability of EPB was improved after silica encapsulation. Above all, the optimum TEOS dosage was $0.8: 1$ (mass ratio of TEOS to PB).

\section{$3.2 \quad$ FT-IR analysis}

After encapsulated silica, the EPB was easy to be functionalized by silane coupling agent, therefore, the reactive pigment composite particles could be easily prepared by grafting MPS onto EPB. ${ }^{27,28}$ The FT-IR spectra of PB, EPB and RPB were presented in Fig. 4 to confirm the successful modification of Phthalocyanine Blue by silica and MPS. As seen in Fig. 4(b) and (c), the characteristic peaks at $813 \mathrm{~cm}^{-1}$ corresponded to the $\mathrm{Si}-$ O-Si stretching vibration absorption peak. In comparison with the spectra of the PB and EPB, the absorption peaks at 1717 and $1636 \mathrm{~cm}^{-1}$ appeared in the spectra of the RPB, which referred to $\mathrm{C}=\mathrm{O}$ and the $\mathrm{C}=\mathrm{C}$ stretching vibration absorption peak,

Table 4 Particle size and PDI of EPB with different TEOS

\begin{tabular}{lcccc}
\hline & $0.6: 1$ & $0.8: 1$ & $1: 1$ & $1.2: 1$ \\
\hline Z-Aver & 209.6 & 196.1 & 222.0 & 226.7 \\
PDI & 0.222 & 0.201 & 0.209 & 0.314
\end{tabular}

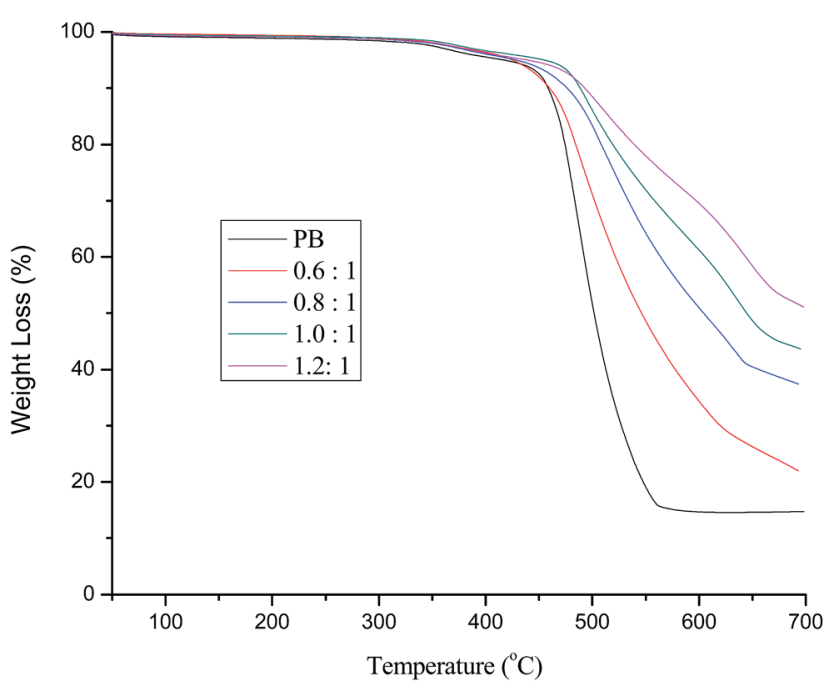

Fig. 2 TGA curves of PB and EPB with different TEOS.

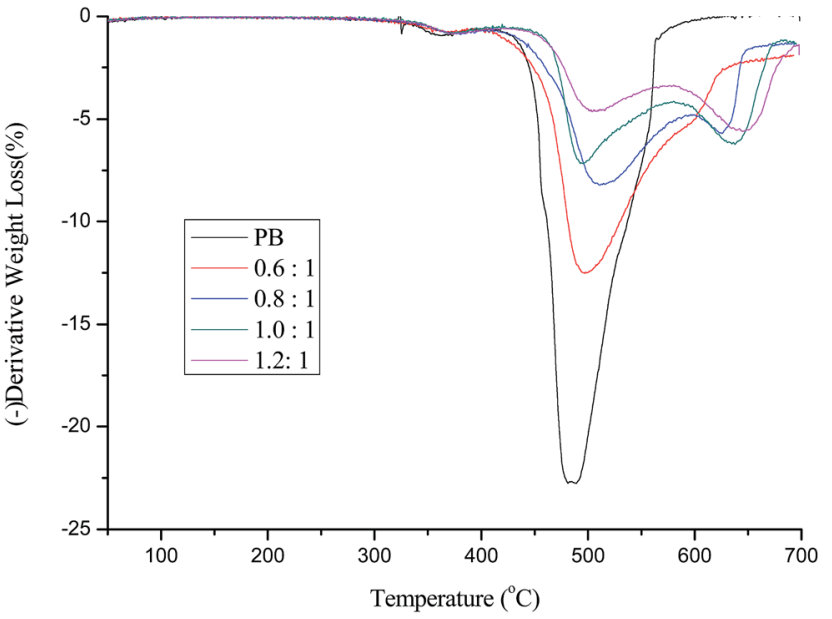

Fig. 3 Differential of the TGA cures in Fig. 2.

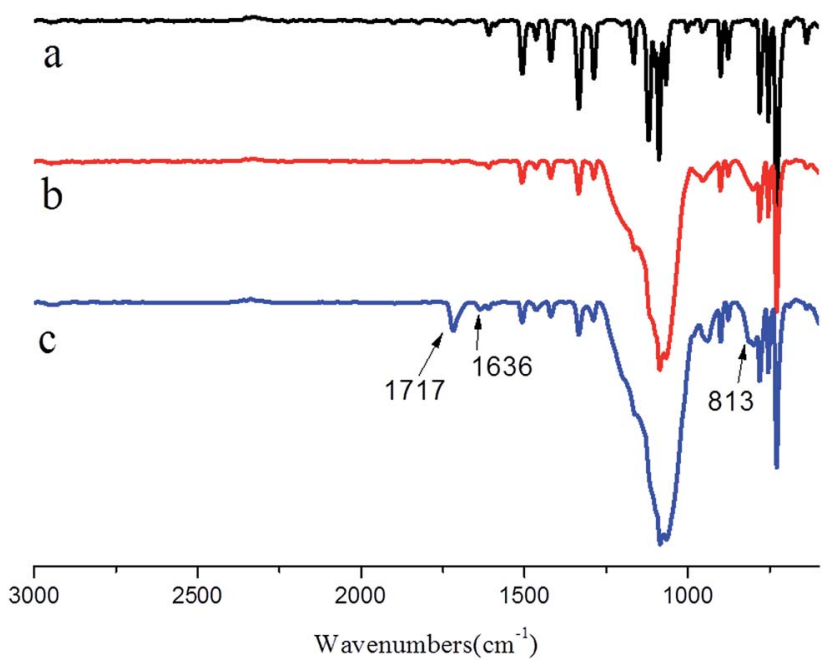

Fig. 4 FT-IR spectra of organic pigment (a: PB, b: EPB, c: RPB).

respectively. Therefore, silica and $\mathrm{C}=\mathrm{C}$ group were introduced onto the surface of PB.

\subsection{TEM and EDS analyses}

The morphologies of PB, EPB and RPB were characterized by TEM microscopy as shown in Fig. 5. Compared with the PB (Fig. 5a), the relatively much darker surfaces could be observed in the EPB and RPB, which indicated that the surfaces of organic pigment were encapsulated with silica. ${ }^{21}$ The presence of silica could be further confirmed by the following EDS analysis.

Fig. 6 showed the atomic compositions of PB, EPB and RPB. It could be seen that, from Fig. 6, there was no presence of atomic silica on the $\mathrm{PB}$, while obvious presence of atomic silica on both the EPB and RPB, indicated by the peaks at $1.8 \mathrm{keV}$ on Fig. 6(b) and (c).

Further observation of TEM images, it seems that there were no $\mathrm{SiO}_{2}$ particles presented on the pigment surface on Fig. 5(b) and (c) though EDS analysis has confirmed the presence of 

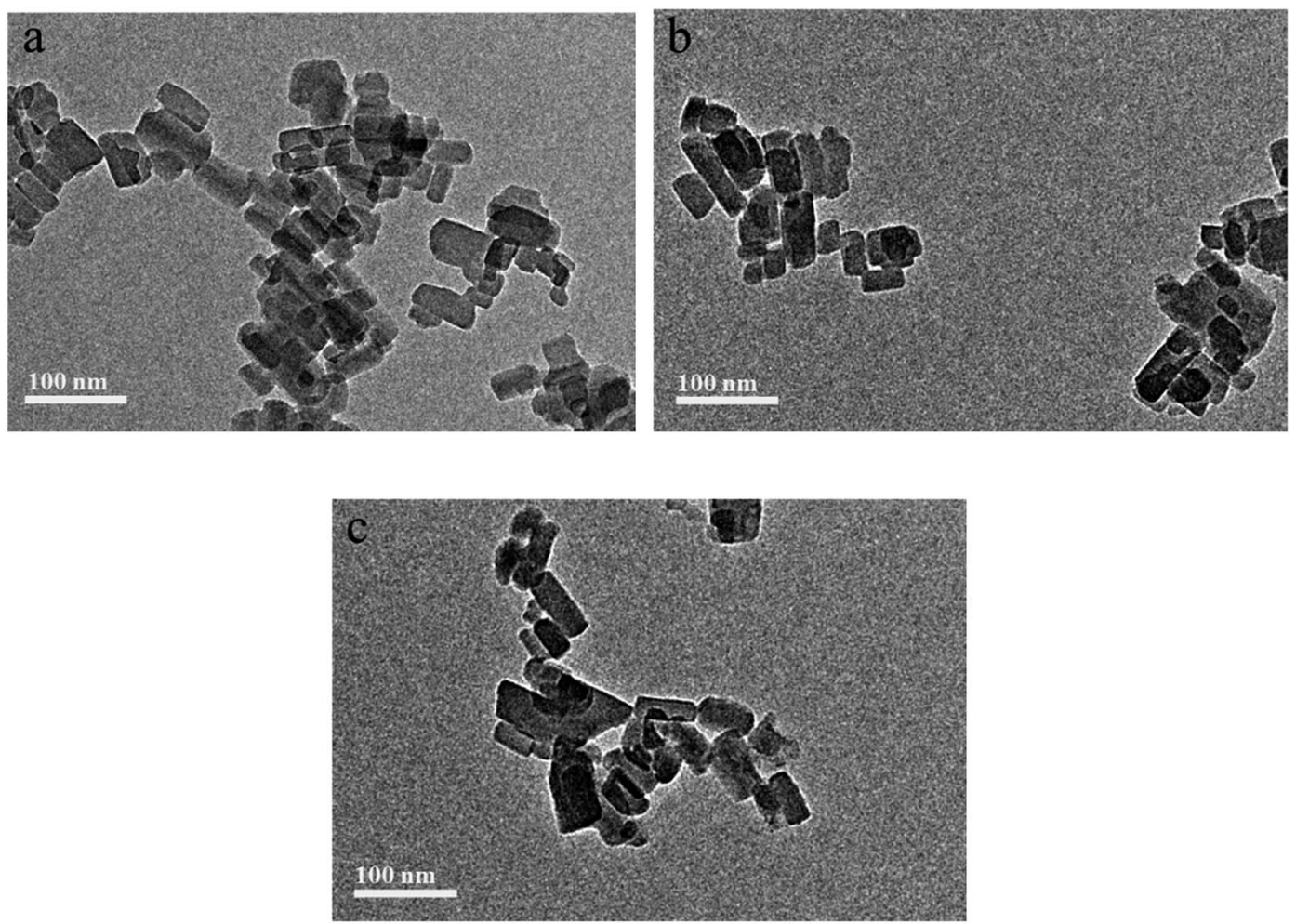

Fig. 5 TEM images of organic pigment (a: PB, b: EPB, c: RPB).
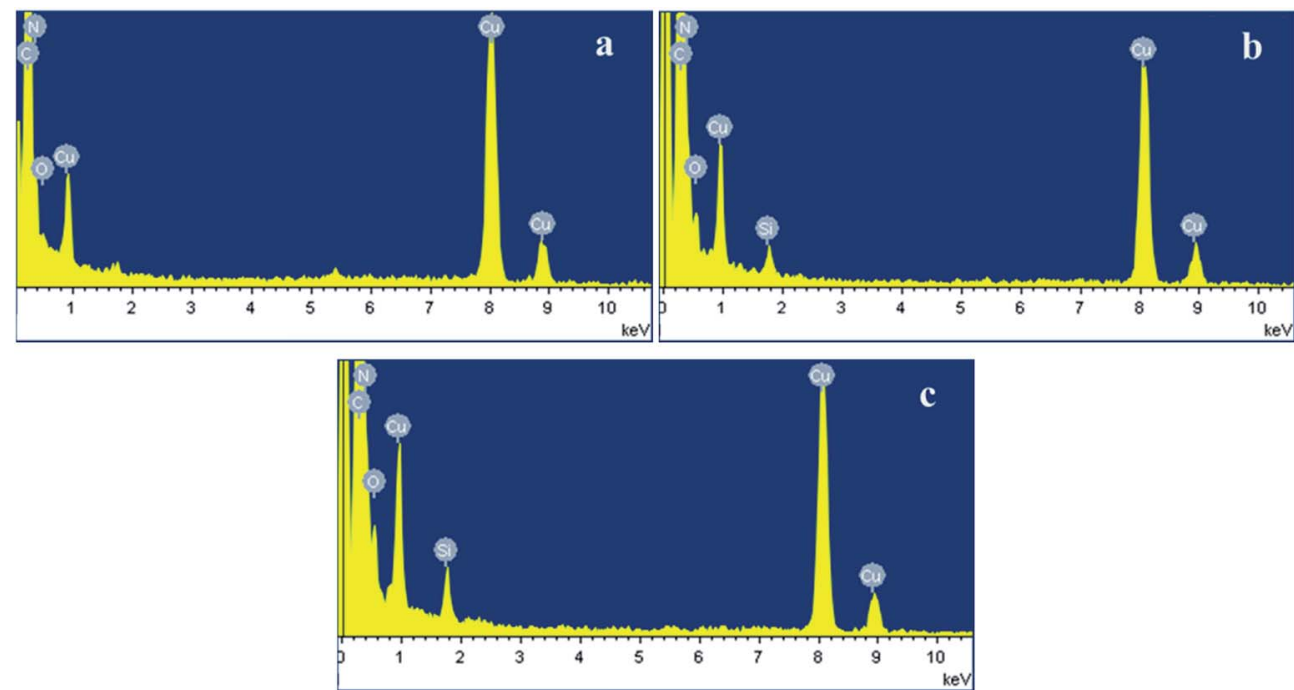

Fig. 6 The atomic compositions of PB, EPB and RPB (a: PB, b: EPB, c: RPB).

silica element. This might be explained by the formation of silica film on the pigment surface during the sol-gel treatment, rather than the formation of $\mathrm{SiO}_{2}$ particles.

\subsection{Contact angle of organic pigment}

The wetting of water on the solid is dependent on the relationship between the interfacial tensions. The contact angle $(\theta)$ between the water droplet and the given surface can be used to represent the hydrophobicity-hydrophobicity. The influences of modification on the water contact angle of organic pigment are shown in Fig. 7. With regard to Fig. 7(a), it was observed that the water contact angle of $\mathrm{PB}$ was $115.95^{\circ}$. However, the water contact angle of EPB was $51.16^{\circ}$, it implied that the hydrophilic of EPB was improved due to the silica encapsulated on the surface of organic pigment. The water contact angle of RPB was increased after grafting MPS. The reaction between MPS and $-\mathrm{OH}$ on the surface of $\mathrm{EPB}$ was occurred, and $\mathrm{C}=\mathrm{C}$ groups 


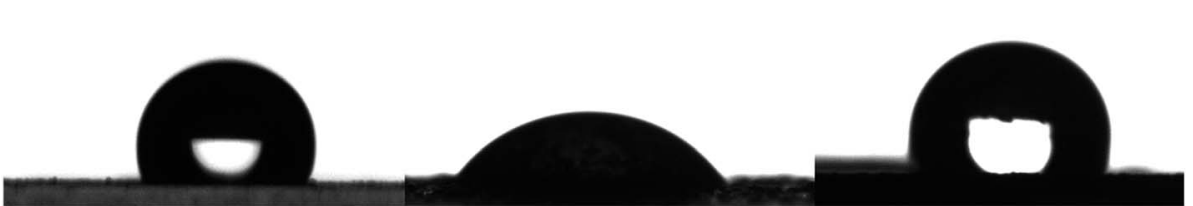

Fig. 7 Contact angles of water droplets on the surface of organic pigment (a: PB, b: EPB, c: RPB).

replaced-OH groups. As a result, the hydrophobicity of RPB was close to the PB.

\subsection{Color performance of modified organic pigment particles}

Fig. 8 displays the influences of modification on the color characteristic of the organic pigment. The color characteristic of the organic pigment was evaluated by examining the changes of the visible diffuse reflectance spectra. In the spectra, the peak locations of PB, EPB and corresponding RPB were almost the same. The RPB kept similar hues to the PB, which could be proved by the appearance of the pigments, as shown in Fig. 8. Therefore, there had little influence of modification on the color performance of the organic pigments.

\subsection{Particle size and dispersion stability analysis}

The particle size distribution of PB and RPB were analyzed by a Nano Particle Analyzer. The results were plotted in Fig. 9. The particle size distribution of RPB was similar to PB. It implied that the average particle size of RPB had little change. The reasons might be that a thin and homogeneous film was formed on the surfaces of the organic pigment particles, and the encapsulation and grafting had little influence on the particle size. Fig. 10 shows the dispersion stability of PB and RPB in

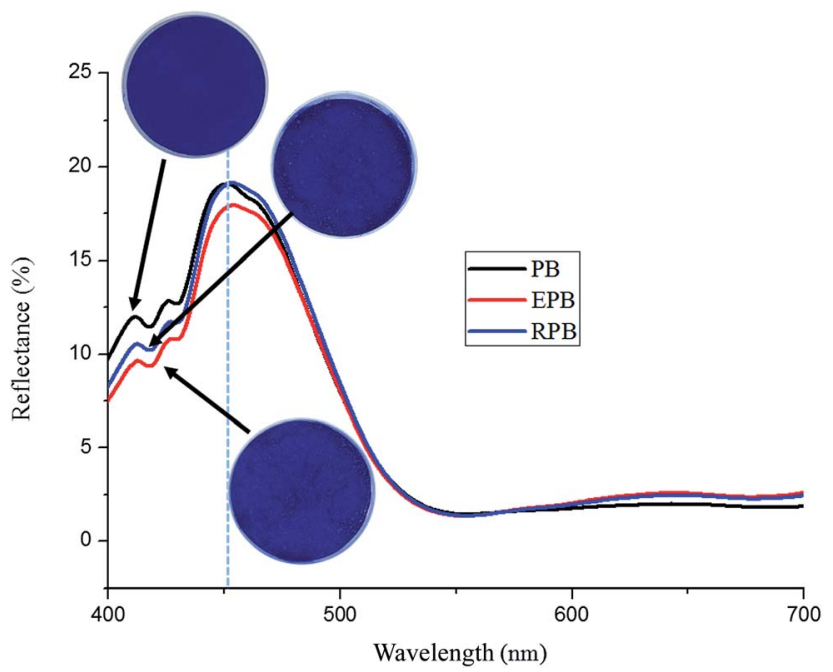

Fig. 8 Reflection spectra of organic pigment.
HEA. After one week standing, significant stratification was found in the PB dispersion. In contrast, there was almost no stratification phenomenon appeared in the RPB dispersion, which indicated that the dispersion stability was improved after modification. The reason was that an outward extension of the organic chain was formed on the surface of RPB which acted as a steric hindrance.

\subsection{The surface tension of inks analysis}

Surface tension is defined as the force per unit length that acts across any line in a surface, tending to pull the surface closed.

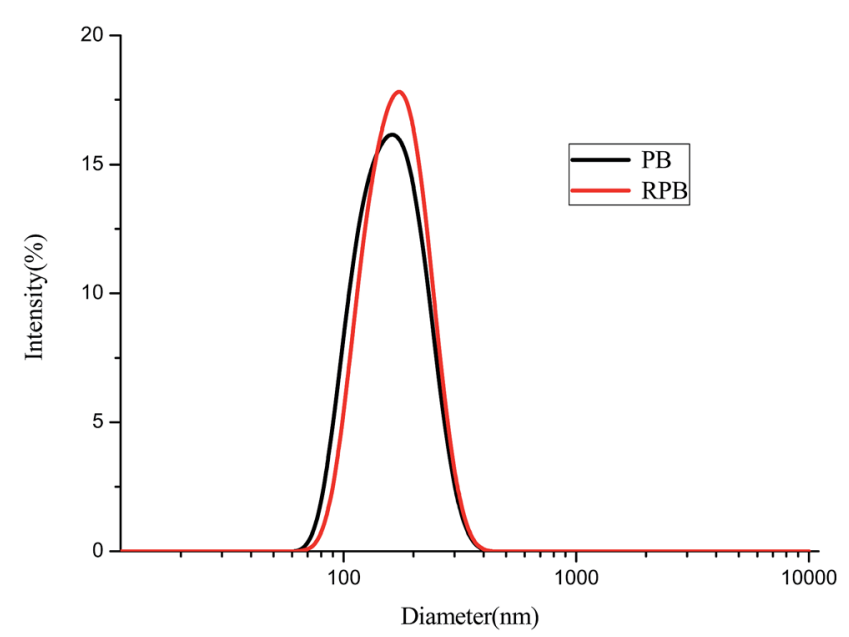

Fig. 9 The particle size of PB and RPB.

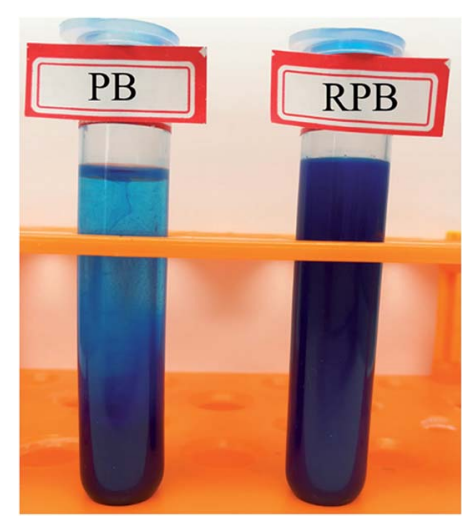

Fig. 10 The dispersion stability of PB and RPB in HEA. 

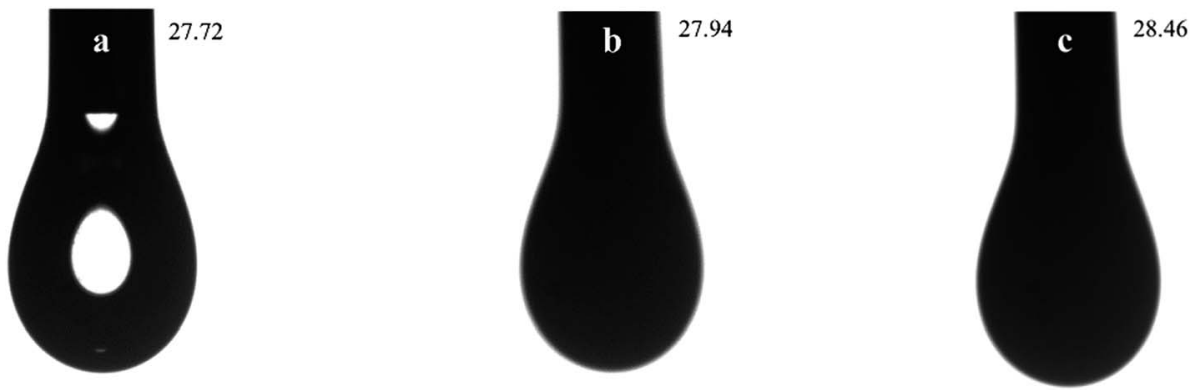

Fig. 11 Surface tensions of inks (a: ink without pigment, b: ink with PB, c: ink with RPB).

Surface tension is calculated using the forces experienced by the balance as the probe interacts with the surface of the liquid. Fig. 11 showed the tensions of different inks. It was found that the surface tension of inks had little change after the addition of PB and RPB. Furthermore, the surface tensions of inks with PB and RPB were almost the same. It suggested that the modification of organic pigment had little effect on the surface tension of inks.

\subsection{Photo-polymerization performance of blue light cured inks}

Photo-polymerization performances of blue light cured inks are presented in Fig. 12. Compared to the blank polymerization system, the photo-polymerization performance of the blue light cured inks declined after the addition of PB or RPB, resulting from the physical shielding effect of pigment particle. The blue light cured ink with RPB had better performances than ink with $\mathrm{PB}$. The reason was that the concentration of double bonds in inks increased after modified. Therefore, the photopolymerization performance of the blue light cured ink with RPB was improved.

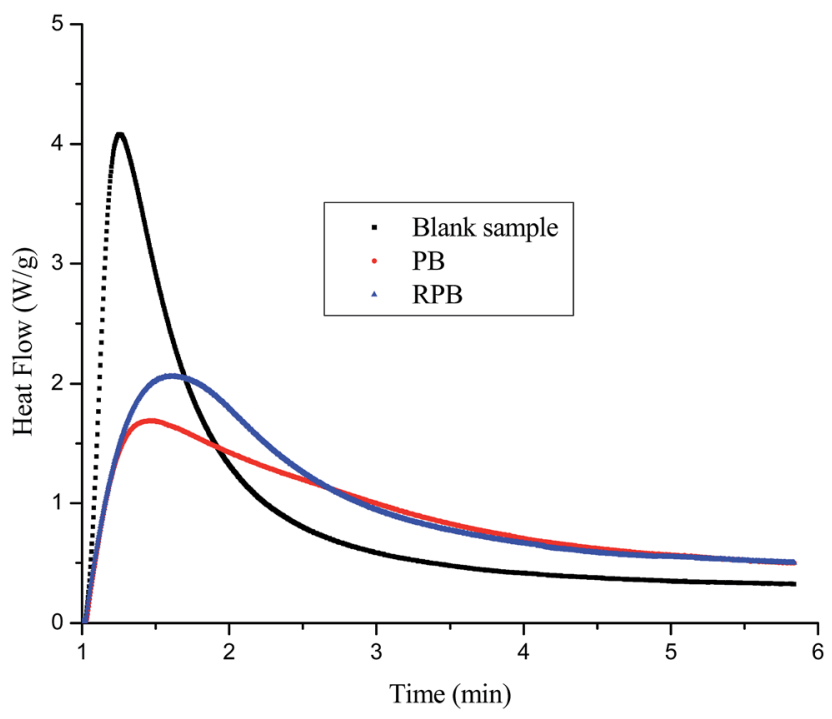

Fig. 12 The polymerization rate of the blue light curable inks.

\subsection{Printing performance}

Table 5 shows the crock fastness of the printed fabrics. It was clearly seen that the crock colorfastness of the printed fabric with RPB was improved. This was probably because the double bonds existed in RPB. The double bonds could participate in the copolymerization of oligomers and monomers, achieving a firm anchorage of pigment particles in the cross-linked polymer network. Fig. 13 showed the stress-strain curves of the cured films. It was found that the elongation at break and breaking strength of cured films were declined after the addition of organic pigment. However, the elongation at break and breaking strength of cured films with RPB were better than PB, which implied that the double bonds introduced in RPB had taken part in the copolymerization of oligomers and monomers.

Table 6 displayed the handle of unprinted and printed fabric. The handle of fabric includes stiffness, smoothness and softness tested by PhabrOmeter fabric style instrument. The

Table 5 Colorfastness to crocking of the printed fabrics with PB or RPB

\begin{tabular}{lll}
\hline & Dry fastness & Wet fastness \\
\hline PB & 3 & 3.5 \\
RPB & 4 & 4.5
\end{tabular}

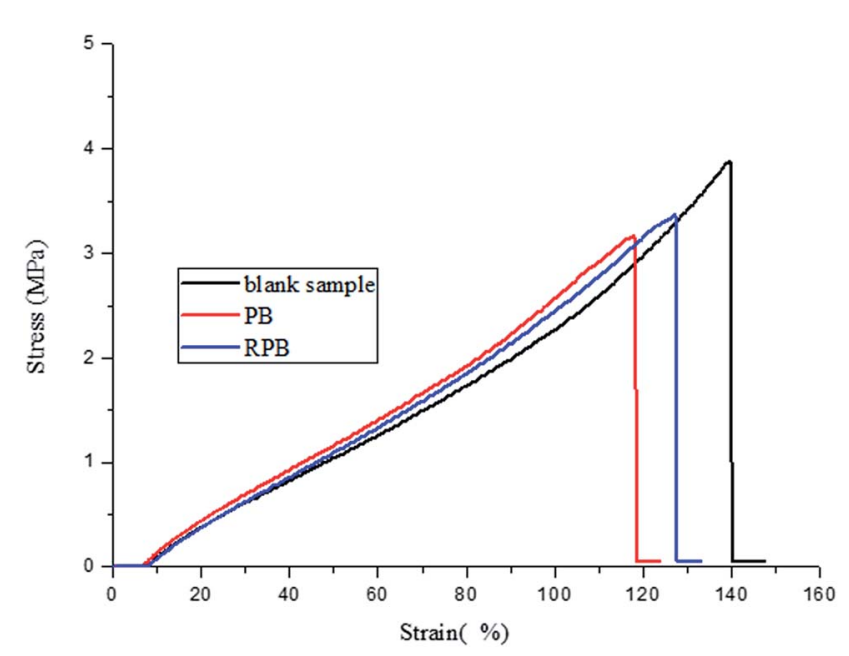

Fig. 13 Stress-strain curves of the cured films. 
Table 6 The handle of the printed fabrics with PB and RPB

\begin{tabular}{llll}
\hline & $\begin{array}{l}\text { Stiffness } \\
\text { score }\end{array}$ & Softness score & $\begin{array}{l}\text { Smoothness } \\
\text { score }\end{array}$ \\
\hline Blank sample & 57.74 & 71.08 & 84.35 \\
PB & 58.31 & 68.82 & 84.69 \\
RPB & 58.84 & 68.46 & 84.74
\end{tabular}

lower the stiffness value is, the greater the smoothness and softness value are, the better the hand feeling. Table 6 showed the stiffness, smoothness and softness of unprinted and printed fabric had little change. It indicated that blue-light curable digital inkjet printing of textiles using RPB had little negative effect on the handle of fabric.

\section{Conclusions}

EPB was obtained by silica encapsulation via sol-gel methods, and the optimized encapsulation conditions were as follows: the dosage of deionized water was $1: 16\left(V_{\text {water }} / V_{\text {ethanol }}\right)$, the $\mathrm{pH}$ value was 10 and the TEOS dosage was $0.8: 1$ (mass ratio of TEOS to PB). The RPB was prepared by grafting 3-(trimethoxysilyl)propyl methacrylate onto EPB. TEM analysis showed that the surfaces of organic pigment were encapsulated with silica, and FTIR analysis indicated that MPS was grafted on the EPB surface as expected. The particle size and hydrophilicity of RPB were similar to those of the PB. For the application properties of blue light curable pigment inks prepared using RPB, Photo-DSC analysis showed a markedly increased polymerization rate, and physical property measurements of printed fabrics showed an improved colorfastness toward crocking, and little negative influence on fabric handle as well.

\section{Acknowledgements}

This work was financially supported by the National Natural Science Foundation of China (grant number 51273180), and Zhejiang Provincial Top Key Academic Discipline of Chemical Engineering and Technology of Zhejiang Sci-Tech University (grant number CETT2015003).

\section{References}

1 D. J. Tyler, Textile Digital Printing Technologies, Text. Prog., 2005, 37(4), 1-65.

2 S. Leelajariyakul, H. Noguchi and S. Kiatkamjornwong, Surface-modified and micro-encapsulated pigmented inks for ink jet printing on textile fabrics, Prog. Org. Coat., 2008, 62(2), 145-161.

3 M. M. Marie, Y. H. El-Hamaky, D. Maamoun, et al., Pigment Ink Formulation for Inkjet Printing of Different Textile Materials, Int. J. Innovation Appl. Stud., 2013, 4(1), 239-247.

4 S. Mhetre and W. C. P. Radhakrishnaiah, On the relationship between ink-jet printing quality of pigment ink and the spreading behavior of ink drops, J. Text. Inst., 2010, 101(5), 423-430.
5 B. Neral, S. Šostar-Turk and B. Vončina, Properties of UVcured pigment prints on textile fabric, Dyes Pigm., 2006, 68(2-3), 143-150.

6 J. Shao, Y. Huang and Q. Fan, Visible light initiating systems for photopolymerization: status, development and challenges, Polym. Chem., 2014, 5(14), 4195-4210.

7 Y. Huang, B. Cao, C. Xu, et al., Synthesis process control and property evaluation of a low-viscosity urethane acrylate oligomer for blue light curable ink of textile digital printing, Text. Res. J., 2014, 85(7), 759-767.

8 Y. Huang, H. Zhang, Y. Meng, et al., Study on Flexible Polymerization System of Blue Light Curing Ink for Textile Digital Inkjet Printing, Journal of Zhejiang Sci-Tech University, 2016, 35(1), 9-16.

9 X. Zhou, J. Zhang, X. Li, et al., Synthesis of core-shell acrylicpolyurethane hybrid latex as binder of aqueous pigment inks for digital inkjet printing, Prog. Nat. Sci., 2012, 22(1), 71-78.

10 S. Fu, L. Zhang, A. Tian, et al., Preparation of a Novel Colorant with Branched Poly(styrene-alt-maleic anhydride) for Textile Printing, Ind. Eng. Chem. Res., 2014, 53(24), 10007-10014.

11 C. Fang, X. Zhou, Q. Yu, et al., Synthesis and characterization of low crystalline waterborne polyurethane for potential application in water-based ink binder, Prog. Org. Coat., 2014, 77(1), 61-71.

12 Y. Guan, B. Tawiah, L. Zhang, et al., Preparation of UV-cured pigment/latex dispersion for textile inkjet printing, Colloids Surf., A, 2014, 462, 90-98.

13 O. A. Hakeim, A. A. Arafa, M. K. Zahran, et al., UV-curable encapsulation of surface-Modified organic pigments for inkjet printing of textiles, Colloids Surf., A, 2014, 447(447), 172-182.

14 C. H. Xue, M. M. Shi, H. Z. Chen, et al., Preparation and application of nanoscale microemulsion as binder for fabric inkjet printing, Colloids Surf., A, 2006, 287(1-3), 147152.

15 S. Fu, D. Wang, C. Du, et al., Improve color fastness of inkjet printing textiles using pigment ink, J. Text. Res., 2015, 36(02), 141-147.

16 S. Fu, C. Du, M. Zhang, et al., Preparation and properties of polymer-encapsulated phthalocyanine blue pigment via emulsion polymerization, Prog. Org. Coat., 2012, 73(2), 149-154.

17 O. A. Hakeim, Q. Fan and Y. K. Kim, Encapsulation of Pigment Red 122 into UV-curable resins via a miniemulsion technique, Pigm. Resin Technol., 2010, 39(1), 3-8.

$18 \mathrm{~S} . \mathrm{Fu}$ and $\mathrm{K}$. Fang, Properties of waterborne nanoscale pigment red 122 dispersion prepared by phase separation method, J. Appl. Polym. Sci., 2008, 108(6), 3968-3972.

19 A. A. Haroun, H. A. Diab and O. A. Hakeim, Cellulosic fabrics printing with multifunctional encapsulated phthalocyanine pigment blue using phase separation method, Carbohydr. Polym., 2016, 146, 102-108.

20 J. Yuan, W. Xing, G. Gu, et al., The properties of organic pigment encapsulated with nano-silica via layer-by-layer assembly technique, Dyes Pigm., 2008, 76(2), 463-469. 
21 J. Yuan, S. Zhou, G. Gu, et al., Encapsulation of organic pigment particles with silica via sol-gel process, J. Sol-Gel Sci. Technol., 2005, 36(3), 265-274.

22 B. Xiao, H. Wu and S. Guo, Regulating the properties of C.I. Pigment Red 170 by surface modification via, hydrous alumina, Dyes Pigm., 2016, 127, 87-93.

23 C. Wang, L. Wang, Y. Huang, et al., Preparation and characterization of Phthalocyanine Blue encapsulated with silane coupling agent for blue light curable inkjet printing of textiles, Dyes Pigm., 2017, 139, 453-459.

24 J. Yuan, S. Zhou, B. You and L. Wu, Organic pigment particles coated with colloidal Nano-Silica particles via layer-by-layer assembly, Chem. Mater., 2005, 17, 3587-3594.
25 B. Zhang, Z. Zhang, X. Fei, et al., Preparation and properties of C.I. Pigment Red 170 modified with silica fume, Pigm. Resin Technol., 2016, 45(3), 141-148.

26 S. Kango, S. Kalia, A. Celli, et al., Surface modification of inorganic nanoparticles for development of organicinorganic nanocomposites-A review, Prog. Polym. Sci., 2013, 38(8), 1232-1261.

27 Z. Wen, Y. Feng, X. Li, et al., Surface modification of organic pigment particles for microencapsulated electrophoretic displays, Dyes Pigm., 2012, 92(1), 554-562.

28 Y. Li, Q. Guo, Z. Wang, et al., Fabrication of tri-layer structured $\mathrm{CdS} / \mathrm{SiO}_{2} /$ sulfonated PS composite sphere and its photocatalytic behavior, Colloids Surf., A, 2015, 486, 106-113. 